\section{REVISTA}

Actualidades Investigativas en Educación http://revista.inie.ucr.ac.cr/

ISSN 1409-4703

\title{
MÓDULO Y DESARROLLO DE COMPETENCIA: ORIGEN DE UNA CONCEPCIÓN DIFERENTE
}

MODULE AND COMPETENCE DEVELOPMENT: THE ORIGIN OF A DIFFERET CONCEPTION

\section{Volumen 15, Número 3}

Setiembre - Diciembre

pp.1-14

\section{Este número se publicó el $1^{\circ}$ de setiembre de 2015}

DOI: http://dx.doi.org/10.15517/aie.v15i3.21073

Berta Margarita González Rivero

Revista indizada en REDALYC, SCIELO

Revista distribuida en las bases de datos:

CATÁLOGO DE LATINDEX, IRESIE, CLASE, DIALNET, DOAJ, E-REVIST@S, SHERPA/ROMEO, QUALIS, MIAR

Revista registrada en los directorios:

ULRICH'S, REDIE, RINACE, OEI, MAESTROTECA, PREAL, CLACSO 


\title{
MÓDULO Y DESARROLLO DE COMPETENCIA: ORIGEN DE UNA CONCEPCIÓN DIFERENTE \\ MODULE AND COMPETENCE DEVELOPMENT: THE ORIGIN OF A DIFFERET CONCEPTION
}

\section{Berta Margarita González Rivero ${ }^{1}$}

\begin{abstract}
Resumen: El ensayo tiene como objetivo exponer las reflexiones acerca de la nueva concepción de un componente curricular denominado módulo de enseñanza y su vínculo con la competencia profesional. Se basa en la aplicación de la teoría del enfoque histórico cultural y en la metodología del análisis estructural-funcional de la actividad a la educación superior. Se expone una breve descripción del procedimiento empleado para determinar los módulos y el surgimiento de la competencia profesional en el proceso de diseño curricular de una carrera universitaria. La concepción de estos componentes (módulo y competencia profesional) difiere de las conocidas hasta el presente, las cuales han sido cuestionadas por diferentes autores. Se concibe el módulo como una unidad estructural y funcional dirigida a la formación de competencias en el profesional. En estas se incluye como elemento central el modo de actuación profesional. La nueva concepción del módulo y de la competencia profesional obtenida cambia, no solo en sus esencias, sino que modifica también los aspectos laborales e investigativos integrados en ellas La utilización de una base científica para la determinación de componentes curriculares permitió llegar a nuevas y sólidas concepciones sobre dichos componentes.
\end{abstract}

Palabras clave: CURRÍCULO, PLAN DE ESTUDIOS, ENSEÑANZA MODULAR, FORMACIÓN POR COMPETENCIA, EDUCACIÓN SUPERIOR, CUBA

\begin{abstract}
The essay has the objective of expose ideas about a new view of a curricula component named module and the join with the professional competence. . It was based in cultural historic theory with a theoretical methodoly of analyze the activity. It show a brief description of the procedures utilized in order to determine modules and the professional competence in the process of university curricula design. The idea of those components (module and professional competence) is different of others that exists, and that are analyzed by others authors. The module in this conception is a structural and functional educational unit oriented to develop professional competence. Its central component is the professional mode of actuation, but it is not the unique. This new view of module and professional competence change their essences and other aspects that are included. The use of scientific theory in order to meet curricular components conduced to new and hard conceptions of them.
\end{abstract}

Key words: CURRICULUM, STUDY PLAN, MODULE EDUCATION, PROFESSIONAL COMPETENCE, MODE OF ACTUATION, COMPETENCE FORMATION, HIGH SCHOOL, CUBA

\footnotetext{
Profesora e Investigadora Titular del Centro Para el Perfeccionamiento de la Educación Superior (CEPES), Universidad de La Habana, Cuba. Doctora en Ciencias Psicológica. Presidenta de la Asociación de Pedagogos de Cuba en la Universidad de La Habana.
}

Dirección electrónica: berta@cepes.uh.cu

Ensayo recibido: $1^{\circ}$ de noviembre, 2014

Enviado a corrección: 23 de enero, 2015

Aprobado: 13 de julio, 2015 


\section{Introducción}

Los Centros de Educación Superior tienen como una de las tareas el perfeccionamiento de sus currículos para hacerlos más adecuados a las demandas. En muchas ocasiones, esta tarea se realiza sin cambios trascendentales debido a las limitaciones teóricas y metodológicas.

Muchos son los intentos de hacer el viraje hacia el educando. Por supuesto, no quiere decir que no se atiendan los conocimientos, por cuanto es imposible el dominio de cualquier profesión sin ellos. No obstante, en el mundo de hoy, es más importante dotar al educando de las esencias y formarlo como persona.

Las bases para lograr un diseño curricular orientado al educando pueden encontrarse en la teoría del enfoque histórico cultural que llevada a la enseñanza, plantea algunas exigencias tales como que se explicite el currículo oculto, se tome en cuenta la actividad y la comunicación para la educación, la concepción social del aprendizaje, la importancia de los mediadores y el educando como centro de la educación.

Esta teoría que fue descubierta y desarrollada por Vigotski (1987), quien tiene el mérito de haber identificado el origen social de las funciones psíquicas superiores, sirve de base a una enseñanza desarrolladora. En ésta se aplican sus categorías y principios

El análisis del Currículum como un todo y de su diseño como un medio o estrategia para abordar su perfeccionamiento juega un papel fundamental para el planteamiento de cualquier investigación en torno a la transformación del proceso docente educativo. Una forma de materializar esto se encuentra en trabajar el currículo como

un proyecto de formación y un proceso de realización a través de una serie estructurada y ordenada de contenidos y experiencias de aprendizaje, articulados en forma de propuesta político-educativa que propugnan diversos sectores sociales interesados en un tipo de educación particular, con la finalidad de producir aprendizajes que se traduzcan en formas de pensar, sentir, valorar y actuar frente a los problemas complejos que plantea la vida social y laboral en un país determinado. (Sanz, 2003, p. 14)

Las transformaciones que requieren los currículos no pueden ser formales ni externas, deben estar sustentadas en una teoría que dirija la formación del hombre, con un criterio histórico y contextual. 
En el proceso de diseño curricular para la Educación Superior, basado en esta teoría, se utilizó un método teórico previsto en dicha teoría. El método utilizado denominado análisis estructural y funcional de la actividad basado en el enfoque histórico cultural, permitió llegar a identificar un componente nuevo. En el presente ensayo se presenta de manera sintética la metodología seguida así como la identificación de tal componente. Solamente se expone la aplicación de dicha metodología para el tratamiento de los contenidos específicos de la profesión, no así todo el proceso de diseño curricular que forma parte de una monografía en proceso de publicación.

Este componente lo constituye el módulo. Él es producto de la aplicación de la teoría de la actividad propuesta por Vigotski y desarrollada por A.N. Leontiev (1981). Precisamente por el método utilizado y por no asumirse las conceptualizaciones existentes, constituye un componente novedoso y diferente a los demás sistemas modulares.

\section{La enseñanza modular}

Resultan insuficientes aún los trabajos que abordan la relación entre el diseño curricular y la formación de habilidades, pues estas últimas se estudian más desde el punto de vista de la didáctica que desde el punto de vista del diseño del currículo. La mirada desde el diseño del propio currículo aporta una visión más acorde al profesional, principalmente, en lo que se refiere a la formación de habilidades profesionales generales y modo de actuación profesional que no pueden ser desarrollados en el marco de una o pocas asignaturas.

Un aporte significativo en la comprensión de cómo se forma la habilidad en el proceso docente educativo es el realizado por P. Ya Galperin (1986), cuando señala que en la línea fundamental de cambios de la formación por etapas de las acciones mentales, la acción varía en tres direcciones: por el grado de generalización, de abreviación y de asimilación de dichas acciones.

La relación entre diseño curricular y formación de habilidades también es tratada en trabajos de Álvarez de Zayas (1988), en los cuales indica que en el proceso docente educativo desarrollado para la formación de habilidades profesionales generales, es posible diferenciar tres componentes esenciales atendiendo al tipo de actividad que desarrolla el estudiante en ese proceso y que su organización y dinámica le permite la apropiación de los modos de actuación profesional. Estos componentes son de carácter académico, laboral e investigativo. 
Se establece, de esta forma, la necesidad de la presencia de ellos como referentes generales para la estructuración de todo un Plan de Estudio, a partir de la necesidad de formación de un determinado tipo de habilidades. Dicho autor enlaza dos aspectos que, generalmente, no se enfocan de una manera tan directa, pues a un mismo diseño curricular puede corresponder más de un enfoque didáctico para la formación de determinadas habilidades.

Esta relación tiene mayor importancia en el caso de las habilidades profesionales generales que no se limitan a los problemas más específicos de una profesión, sino a aquellos aspectos más generales y característicos de la conducta de un profesional que se deben formar a través de todo el currículo.

Debe señalarse que para lograr la integración de los tres componentes (académico, laboral e investigativo) la estructura de una asignatura, en un currículo disciplinar, no siempre se presta adecuadamente para ello y se han buscado diversas soluciones, planes directores, disciplinas principales integradoras, jerarquía de asignaturas, a través de prácticas laborales integrales, proyectos de curso y otros. Todas ellas con aciertos y dificultades. En realidad, en esas propuestas se introducen principios de la enseñanza modular que propician una reestructuración de los contenidos y los métodos de enseñanza pero que, en ocasiones, no se trabajan a profundidad, sino que se adicionan como "parches" y no alcanzan el nivel de sistematicidad e integración requeridos.

Una alternativa a la estructuración por disciplina o asignaturas, ha surgido hace unos años como globalizadora o integradora del currículo. Este modelo ha sido puesto en práctica en la enseñanza de pregrado en países de América Latina (México, Ecuador).

Esta estructuración tiene sus antecedentes pedagógicos en el movimiento de la Escuela Nueva (activa), en la cual surgen las primeras propuestas globalizadoras a través de los llamados centros de interés de Decroly, centrada en la unidad de la ciencia y la realidad (Llanio, 1997). La organización del conocimiento en unidades de enseñanza, módulos o bloques se generó a partir del modelo desarrollado por la Escuela de Medicina de la Universidad de Western Reserve.

Fue en 1974 que considerada una innovación, se propone la organización de un sistema de enseñanza aprendizaje llamado Sistema Modular (SM), adoptado por la Universidad Autónoma Metropolitana, campus Xochimilco de México. La singularidad de este sistema es diseñar tanto el plan como los programas de estudios, en torno a problemas de la realidad y no en objetivos y contenidos propios de asignaturas académicas. Según Payán y 
Guerra (2015) el alumno es responsable de su aprendizaje y conoce la realidad mediante objetos y no de forma disciplinar.

El modelo educativo que la Unidad Académica de Xochimilco de la Universidad Autónoma Metropolitana adoptó, desde aquél entonces, ha desafiado creencias fuertemente arraigadas en la comunidad académica, pero generó también críticas que en no pocos casos fueron de sus mismos seguidores.

Las modificaciones de fondo que plantea el SM en todos los elementos que conforman la práctica universitaria, consisten en: una redefinición social de las profesiones, una reorientación de los objetivos institucionales hacia los problemas que afectan a los sectores mayoritarios del país, una definición de los perfiles profesionales necesarios para atender tales problemas y nuevas y mejores formas de concebir y operar el sistema de enseñanzaaprendizaje. (Fernández, Páramo y Álvarez, 2011)

La concepción y las experiencias prácticas de este Sistema mostraron las limitaciones que generaba. A la solución de ellas se orientaron las mejoras posteriores. En ese camino se perdieron aciertos y no fueron vencidas las resistencias, lo que no permitió cosechar con certeza los logros que dicha experiencia podía brindar.

Las dos categorías sobre las que se instrumenta toda la organización, formación y desarrollo del currículo en la experiencia mexicana son el objeto de transformación y el problema eje. Al desarrollo y aplicación de estas categorías se dedicó un gran esfuerzo, el cual no en todo momento recibió el reconocimiento necesario.

. El Objeto de Transformación se define como" un enunciado sintético de una situación o problema de la realidad que por sus características de vigencia, relevancia y pertinencia, ha sido incorporado al proceso de enseñanza aprendizaje para el desarrollo de un perfil profesional previamente establecido" (Velasco, R. 1982, p 20, citado por Fernández et al, 2011). El Problema Eje es una expresión contextualizada y empíricamente "capturable" del Objeto de Transformación.

Durante todos estos años, se ha intentado seguir el proceso de desarrollo de este modelo educativo y el panorama que va presentando.

Según exponen Margarita Fernández, Teresa Páramo y Lorena Álvarez (2011, p. 23), En 1991, el Consejo Académico de la Unidad Xochimilco describe esa situación de la siguiente manera... "la experimentación sin control experimental, la innovación efímera, parcial y fragmentaria, sin evaluación ni seguimiento, la degradación de franjas importantes de la organización académica son elementos que han coexistido 
con desarrollos académicos de excelencia, a través de procesos innovadores altamente significativos".

En 2007 se reconoce la aportación del Sistema Modular en un 48\% del total de egresados de la UAM del período 1974-2007, prácticamente la mitad de los egresados de las 3 unidades, el doble de lo alcanzado en promedio por las otras 2 unidades. Sin embargo, las fuerzas internas y del contexto han jugado simultáneamente tanto a favor como en sentido contrario del Sistema Modular (Fernández et al., 2011). Padilla (2012) hace una descripción bastante completa de esta concepción del Sistema modular, llegando a la conclusión de que el tiempo les ha dado la razón. No obstante, apunta que se presenta una pluralidad de formas de entender y practicar la actividad docente, lo que tal vez, ha evitado confrontaciones más agudas.

En los años 80 se introdujo un modelo similar en la Universidad Nacional de Loja, en Ecuador. En ambos modelos hacen énfasis en la organización de los contenidos por un Objeto de Transformación, definido como un problema de la práctica profesional. Su estudio se realiza atendiendo a todos sus ángulos (técnicos, económicos, políticos, etcétera).

En Brasil se aprueba una Ley sobre directrices y bases Nro. 9394/96 que introduce nuevas orientaciones para la educación, entre ellas, para la enseñanza modular. Desde 1977 por decreto federal se organizó la formación técnica media por el sistema modular en este país, para flexibilizar y acercar a la producción la enseñanza. Zíbas (2006) hace una presentación de la evolución de este modelo en este nivel de enseñanza y valora las críticas que ha recibido, no atribuibles a la concepción propiamente modular.

Ya con la denominación de módulos se identificaban cursos independientes realizados en la enseñanza posgraduada, pero empezaba a invadir el nivel de pregrado. Las experiencias más conocidas son las antes mencionadas, de México y Ecuador con una base epistemológica en la teoría piagetiana y con una concepción más integral del currículo..

El concepto "módulo" proviene del campo de la arquitectura y diseño industrial que lo consideran como una unidad autosuficiente y ensamblable. Por esta razón, no aparecen claras sus conceptualizaciones en educación, aunque se traspoló esa imagen a la teoría pedagógica. Por ello, se cuenta en general, con las definiciones de pequeños proyectos que lo han puesto en práctica en sus currículos.

En sentido general, el módulo es la conformación de una especie de unidad a partir de los aportes de diferentes disciplinas en torno a un elemento central que los nuclea. Esta 
organización de la enseñanza, se aparta de la disciplinar, porque no parcela el conocimiento y asume el elemento central en su dinámica e integralidad.

En el análisis que realiza Pansza (s.f.) sobre este tipo de enseñanza, hace una crítica a las conceptualizaciones de esa modalidad que se han divulgado como innovadoras, pero que en muchas de sus aplicaciones no se aleja de la tradicional.

Ha recibido críticas y es incomprendida todavía por muchos docentes. Entre las críticas más importantes están: la integración en torno a problemas de la práctica que envejecen y limitan la amplitud y solidez en la formación; su base epistemológica utilitaria y pragmática; el rompimiento de las fronteras de las diferentes ciencias con el peligro de beneficiar a unas más que a otras; la complejidad de su didáctica; la insuficiente preparación de los profesores para enfrentarla (Díaz, 1993; Pansza, s.f.).

La experiencia cubana que se toma como base de esta exposición llega a resultados favorables en varios años de aplicación. Surge a partir de un diseño curricular elaborado sobre la base de la teoría del enfoque histórico cultural y la teoría de la actividad que define como componente curricular un módulo con una concepción que difiere de las conocidas, por lo que no puede atribuírsele las críticas antes mencionadas.

\section{La determinación de los módulos}

La concepción expuesta no surge de la valoración de los antecedentes de esta enseñanza, sino como consecuencia de un proceso de diseño, con una metodología teórica denominada "análisis funcional-estructural de la actividad", cuyo fundamento está en la teoría materialista dialéctica sobre el concepto actividad utilizado en este caso por Vigotski (1987) y con base científica como se ha expresado antes.

La aplicación de un conjunto de postulados de esta teoría en el diseño curricular conlleva, en una de sus etapas, al análisis de los contenidos directamente vinculados con la profesión de que se trate. El diseño tomó en cuenta los postulados de la teoría del enfoque histórico cultural referidos al principio de la unidad de actividad y conciencia en la de formación de la personalidad, la zona de desarrollo próximo, el aprendizaje de las esencias del conocimiento, así como la integralidad del mismo. (Sanz, 2003).

En correspondencia con esta fundamentación se realizan diferentes análisis que parten desde el modelo de perfil hasta la determinación de los componentes del plan de estudios. La premisa para las decisiones se centró en un conjunto de necesidades formativas 
determinadas con los diferentes métodos utilizados: de expertos, entrevistas y cuestionarios a profesionales en ejercicio.

Con la información obtenida quedó clara la necesidad de formar un profesional competente con dominio de su especialidad en correspondencia con las exigencias de la época, lo que además coincidía con las nuevas tendencias de la formación. Esto plantea una demanda que no fue preestablecida, sino surgida del procesamiento e interpretación de la información como resultado del método aplicado. Esa necesidad se resumía en el dominio de la actividad profesional, de ahí que se fue al análisis de la misma.

Con la utilización de un grupo de expertos bien calificados, se siguieron los pasos de la metodología para analizar la actividad profesional. Conllevó un trabajo riguroso y detallado, al que se le dedicó tiempo. Fueron muy valiosas las reflexiones acerca de lo que los profesionales conceptualizan como lo más necesario para realizar bien la actividad, pero centrándose en el sujeto.

Se siguió rigurosamente la metodología seleccionada. Los pasos realizados y los métodos aplicados se sintetizan en:

- Determinación de las tareas terminales (típicas y frecuentes) de la práctica profesional

- (Método de expertos, métodos prospectivos, entrevistas y observación).

- Determinación del tipo de actividad que permite resolver cada tarea (Método de análisis, deducción e inducción, expertos).

- Análisis estructural y funcional de cada actividad profesional y comparación entre ellas por sus componentes estructurales. (Modelación y comparación).

- Determinación de los modos de actuación particular para cada grupo de actividades y del

- modo de actuación general (Modelación, inducción-deducción).

- Selección de los contenidos (conocimientos, métodos y valores) requeridos para ese modo de actuación. (Modelación, trabajo en equipo, inducción, deducción, análisis, síntesis).

- Determinación de las competencias profesionales. (Análisis y síntesis, modelación).

La organización de los contenidos, después de aplicada la metodología, mostró que no era posible resolver la formación de esa unidad de manera disciplinar por lo que obligó a buscar un componente que no existía en ese momento y fue denominado módulo por acercarse a las características que se le atribuían a este. El propio análisis realizado 
mostraba que no sólo esa unidad era de carácter interdisciplinar, sino que estaba organizada con un núcleo dirigido a la profesión y que todos consideraban que era lo que hacía al profesional competente.

En el análisis de la actividad profesional como categoría vigotskiana, se añadió una reflexión acerca de qué necesitaba el sujeto para realizarla y esto permitió llegar a las estructuras psicológicas necesarias para la realización de esa actividad, la competencia profesional. El hecho de que sean estas estructuras personales las que se conviertan en objetivos educativos justifica la adopción de esa forma organizativa curricular específica que permite un enfoque interdisciplinar (no sólo de conocimientos), integrado y sistémico. La única forma organizativa que puede garantizarlas es el módulo. Pero esta concepción de módulo no se asemeja a las ya conocidas porque no parte de los problemas de la profesión ni tampoco considera el mismo objetivo de formación al que están dirigidos.

Como se ha expuesto, al módulo se llega por el proceso de análisis del modo de actuación profesional, el cual constituye el componente central de las competencias y estas son el núcleo en torno al que se integra el módulo con todos los contenidos (cognoscitivos y afectivos) requeridos para ello. Lo que posibilita llegar a este modo de actuación profesional es el método teórico científico utilizado (Talizina, 1984).

De esta forma, quedan determinadas un conjunto de competencias profesionales esenciales que constituyen el principal contenido profesional de la formación. Ellas tienen un grado elevado de generalidad y garantizan que la formación de la personalidad sea lo esencial.

El proceso seguido hasta aquí, si bien parte de la misma práctica profesional, no queda en ese nivel concreto, no se centra en los problemas, sino que parte de las tareas y se dirige a la actuación del profesional. De ese primer nivel de análisis se dirige a uno más elevado donde se sintetiza, se integra, se va al modo de actuación del profesional para resolver las tareas con el objeto de su profesión o mejor, a aprender a realizar las actividades profesionales que tienen contenidos los problemas actuales y futuros. Lo que es más, se dirige al aprendizaje de las competencias profesionales.

Ellas no sólo tienen un alto nivel de generalidad, sino que son determinadas por las exigencias que pone al sujeto la propia actividad profesional en cuanto a elementos ejecutores y dinámicos. De esta manera, es la orientación hacia el profesional, como educando, hacia la formación de su personalidad, lo que determina los objetivos a alcanzar. 
Los módulos se estructuran con las competencias profesionales. A cada competencia profesional se hace corresponder un módulo de estudio, siendo ella su núcleo rector que va más allá del conocimiento. Cada ciencia tiene que aportar no sólo los contenidos, sino esclarecer los nexos y su papel formativo en la profesión. Po ello, es posible una reorganización de la lógica de ellas, así como la determinación de los núcleos de integración que posibiliten el aprendizaje de la competencia que es el objetivo interdisciplinar. El principio profesional modifica la lógica del contenido de las ciencias, al contextualizarlo.

Estas competencias, por el método utilizado, se corresponden al sector profesional específico. No pueden enlistarse ni intercambiarse ni traspolarse a diferentes profesiones, lo que requeriría la realización del mismo proceso para su determinación. De esta manera, en este caso, no puede hablarse de formación por competencias, como es común en los diseños conocidos, sino aprendizaje de competencias. Estas, como se ha mostrado, no se seleccionan de un listado preestablecido, sino que son particulares (determinadas) de la profesión de que se trate.

Los esfuerzos por los currículos diseñados por competencias profesionales están dirigidos a acercar la formación a la práctica. Esta búsqueda de vinculación entre la teoría y la práctica no puede lograrse sobre la base de criterios empíricos o practicistas sino a partir de un análisis profundo de la teoría que la fundamenta y con la metodología apropiada. El diseño curricular elaborado aunque se orienta a formar competencias profesionales, no se puede incluir en la tradicional formación por competencias donde no está clara la fundamentación teórica y metodológica.

\section{Una concepción diferente de módulo}

La conceptualización del módulo y la competencia profesional desde este nuevo enfoque fue posible al tomar en cuenta una teoría y utilizar un método científico de análisis. Lo novedoso es que muchos conceptos sobre módulo y competencia profesional no surgen de la aplicación de un método teórico mas en este caso es así.

Como se ha mencionado, el elemento integrador que sirve de núcleo interdisciplinar en cada módulo es la competencia profesional. Ni la concepción ocupacional, ni la funcional ni la constructivista pueden explicar adecuadamente la concepción y origen de la competencia profesional, ella tiene que ser determinada en el análisis de la propia actividad profesional con un análisis sistémico. La competencia profesional es una compleja estructuración a la que se llega mediante la enseñanza y la vida, que incluye un conjunto de componentes de 
carácter diferente que permite el funcionamiento holístico y autorregulado, vinculado al buen desempeño en una profesión. La competencia, por tanto, incluye diferentes elementos por lo que no puede reducirse a ninguno de ellos. Asimismo, está sesgada por el carácter de la actividad a la que se orienta y que establece exigencias, pero tampoco puede reducirse a términos de desempeño o resultados. De ninguna forma puede limitarse a una visión enmarcada en el contenido de los cargos o puestos específicos de trabajo, aunque puedan ser su referente (González, 2010).

La competencia se concibe como una estructura personal compleja formada por la confluencia de diferentes elementos, mediante un proceso de aprendizaje en el que se vinculan contenidos teóricos, prácticos y personales. Su componente esencial (no el único) es el modo de actuación profesional particular o general que permite alcanzar los resultados en el conjunto de tareas terminales de la profesión. Este componente curricular no está limitado a los problemas actuales, sino a las tareas típicas y frecuentes que permiten resolver los problemas actuales y futuros. Son identificadas como tareas terminales a partir de su incidencia en el objeto de la profesión. Es conveniente aclarar que son las tareas terminales y no las funciones del cargo. Tomar como premisa las funciones no permitiría organizar su aprendizaje, debido al grado de generalidad que tienen, por lo que son las tareas directamente orientadas al objeto de la profesión las que lo permiten.

Para la determinación de estas tareas se requiere una claridad sobre ese objeto, tanto de su estructura como de sus transformaciones en el actuar del profesional. De esta forma, la concepción del modo de actuación profesional no se orienta a la solución de problemas, lo que le daría un carácter más bien utilitario, sino va a la actuación profesional que cuando es asimilada lleva a la formación de competencias profesionales. Con ello, el profesional será capaz de resolver y de prever problemas propios; así como realizar innovaciones en su campo científico.

La concepción modular presentada exige la confluencia de las diferentes ciencias que se vinculan a la profesión. Requiere la interdisciplinariedad, la cual puede tener diferentes elementos integradores (González, 2013). En este caso lo constituye la competencia profesional.

En esta concepción se concibe el componente laboral o de servicio como parte del propio proceso de aprendizaje y no como un elemento aislado en el currículo. Constituye un momento esencial para la asimilación del modo de actuación. Por ello, el componente laboral 
forma parte consustancial del módulo. Es al mismo tiempo, formador de la competencia y posibilidad de desempeño..

La práctica profesional o laboral contribuye a hacer más sólido y flexible el modo de actuación y es fundamental para la formación de la competencia profesional. En esta idea no se ve aislada la práctica laboral o de servicio, porque ella constituye el momento material del aprendizaje que no concluye ahí. El aprendizaje no se completa con la etapa materializada, sino que debe concluir con la formación de todas las etapas de las acciones mentales (Galperin, 1986).

La formación de habilidades científico-investigativas es otro componente del módulo. Aquí la investigación es concebida fundamentalmente como formativa, con el fin de consolidar la competencia y lograr un mejor desempeño. Con ella se amplía el dominio de la profesión, lo que requiere una adecuada selección de los temas a investigar.

El módulo, en esta concepción, es una unidad de aprendizaje de contenido ejecutor y dinámico. Tiene carácter interdisciplinar y sistémico en el que se integran docencia, investigación y servicio. Su estructuración es con base en las competencias profesionales como núcleo central. Estas tienen una estructura compleja, donde están el modo de actuación profesional que incluye las habilidades, los métodos y las representaciones de tareas y resultados; las capacidades; los valores; las actitudes y motivos.

\section{Conclusiones}

La enseñanza modular ha constituido una alternativa que, a pesar de las críticas recibidas, alumbra el camino hacia el desarrollo. Los antecedentes de este tipo de enseñanza la conciben a partir de problemas de la práctica, estructurada en una unidad de carácter integral y sistémico.

Se conocen experiencias en diferentes países que han intentado incorporar esta forma de enseñanza a la educación de pregrado. Sus bases teóricas, independientemente de las resistencias que produce, provocan que tenga fisuras en lo que debería ser una concepción globalizadora de la enseñanza tan necesaria en estos tiempos y tan a tono con los retos actuales.

No obstante sus críticas, es un encomiable intento de transformar de manera radical las concepciones tradicionales y remover la creatividad de los actores del proceso formativo en la enseñanza de pregrado. 
De este modo, teniendo como base el enfoque histórico cultural y la aplicación de una metodología de análisis teórico para determinar los objetivos educativos se elaboró una concepción modular que se aparta de dichos enfoques. El módulo se concibe como una unidad de contenido ejecutor y dinámico dirigido a la formación de competencias profesionales. En ellas, se encuentra como elemento central el modo de actuación profesional que no tiene una base utilitaria ni pragmática porque va a los procedimientos típicos y generales de la realización de las tareas y actividades profesionales. Para la determinación de las competencias profesionales no se toma, como en otros casos, un listado preelaborado, sino que se realizan diversos pasos metodológicos dirigidos a analizar la actuación del profesional y el carácter de la profesión de que se trate. La metodología garantiza un resultado riguroso y justificado para orientar el diseño del plan de estudios y los diferentes programas. Por otra parte, lleva a la incorporación de componentes curriculares que no están previstos en el pregrado, como los módulos.

Esta metodología creada orienta la formación de un profesional, no, para la solución de los problemas ni de carácter utilitario, sino para su desempeño competente en los problemas actuales y futuros.

\section{Referencias}

Álvarez de Zayas, Carlos. (1988). Fundamentos teóricos de la dirección del proceso de formación del profesional de perfil amplio. Santiago de Cuba: Universidad Central de Las Villas.

Díaz, Frida. (1993). Aproximaciones metodológicas al diseño curricular: hacia una propuesta integral. Revista tecnológica y comunicación Educativos. Recuperado de http://investigacion.ilce.edu. $\mathrm{mx} / \mathrm{stx}$.asp?id=2562

Fernández, Margarita, Páramo, Teresa y Álvarez, Lorena. (2011) El sistema modular en el proceso de enseñanza-aprendizaje la licenciatura de administración: una experiencia de "innovación ordinaria". Cuadernos de Educación y Desarrollo, 3(30). Recuperado de http://www.eumed.net/rev/ced/30/rpa.html

Galperin, P. Ya. (1986). Sobre el método de formación por etapas de las acciones intelectuales. En Antología de la Psicología Pedagogía y de las Edades (pp. 114-117). La Habana, Cuba: Editorial Pueblo y Educación.

González, Berta M. (2010) Competencia y aprendizaje de competencias profesionales. Ponencia presentada en 7 Congreso Internacional de Educación Superior "Universidad 2010", 8-12 de febrero, Cuba. 
González, Berta. (2013). La interdisciplinariedad: un rasgo de la educación contemporánea. Revista Marista de Investigación Educativa (RMIE), $3(4$ y 5), 23-35. Recuperado de www.rmie.maristas.edu.mx

Leontiev, Alexei N. (1981). Actividad, conciencia, personalidad. Cuba: Editorial Pueblo y Educación.

Llanio, Giraldo. (1997). Enfoque Modular en el diseño curricular. La Habana, Cuba: Buró de Investigaciones Pedagógicas de las FAR.

Padilla, Alberto. (2012). El Sistema Modular de enseñanza: una alternativa curricular de educación superior universitaria en México. Revista de Docencia Universitaria, REDU, 10(3), 71-09.

Pansza, Margarita (s.f.) Pedagogía y currículo (fotocopia). Recuperado de https://educacionsuperiorenvenezuela.wikispaces.com/file/view/LIBRO+Pedagog\%C3 \%ADa+y+curriculo.pdf

Payán, Teresita C. y Guerra, Margarita. (2015). La investigación en el sistema modular: una estrategia de enseñanza aprendizaje. Recuperado de http://www.uned.es/congresointer-educacion-intercultural/Grupo discusion 3/38.\%20T.pdf

Sanz, Teresa. (2003). El currículo. Su conceptualización. En Miriam González, Adela Hernández, Herminia Hernández y Teresa Sanz (Coords.), Currículo y formación profesional (pp. 7-25, Capítulo 1). La Habana, Cuba: CEPES, Instituto Politécnico José Antonio Echevarría.

Talizina, Nina F. (1984). Psicología de la enseñanza. Moscú: Editorial Progreso.

Vigotski, Lev S. (1987). Historia del desarrollo de las funciones psíquicas superiores. Cuba: Editorial Científico-Técnica.

Zíbas, Dagmar M.L. (2006). Una visión general de la educación técnica en Brasil. Ponencia presentada en el Encuentro Internacional sobre Enseñanza Técnica-Profesional, Buenos Aires, 6 y 7 de diciembre. Recuperado de http://www.fcc.org.br/conteudosespeciais/difusaoideias/pdf/encontro una vision gener al de la educacion tecnica en brasil.pdf 